\title{
Processo de Validação da Região de Entrada de um Escoamento Laminar em Duto Cilíndrico via
}

\author{
Pinheiro, V. P. ${ }^{1}$; Loeffler, C.F. ${ }^{1}$; Neves, N.S. ${ }^{2}$; Bodart, N.L.O ${ }^{3}$; Melo, L.D.V. ${ }^{3 *}$; \\ Salgado, A.L. ${ }^{3}$
}

1 Programa de Pós-Graduação em Engenharia Mecânica, Universidade Federal do Espírito Santo, Vitória, ES, Brasil.

2 Programa de Pós-Graduação em Engenharia Civil, Universidade Federal do Espírito Santo, Vitória, ES, Brasil.

3 Programa de Iniciação Científica em Métodos Numéricos, Universidade Vila Velha, Vila Velha, ES , Brasil.

* e-mail: lucca1000@gmail.com

\begin{abstract}
Resumo
Há um vasto conjunto de estudos analíticos, numéricos e experimentais na literatura voltados a um entendimento mais consolidado do comportamento da região de entrada de escoamentos nas mais diversas geometrias. Tais estudos são motivados pela extensa aplicação correlata ao fenômeno em vertentes como a indústria de petróleo e gás natural, ramo de siderurgia, produção de energia dentre inúmeras outras. O vigente artigo tem como objetivo a validação da simulação numérica de um escoamento de ar através de duto cilíndrico em regime laminar, feita via programa ANSYS CFX. A investigação numérica releva a presença de picos em regiões próximas a parede da tubulação, no perfil de velocidade axial, como previsto de forma consolidada na literatura. De forma geral, os resultados das simulações mostram boa concordância com diversas referências analíticas e numéricas.
\end{abstract}

Palavras-Chave: Região de Entrada; Processo de Validação; Regime Laminar; Dinâmica dos Fluídos Computacional;

\begin{abstract}
There are a wide range of analytical, numerical and experimental studies in the literature that aims to consolidate understanding of the behavior of the inlet region in the most diverse geometries. Such studies are motivated by the extensive application related to the phenomenon in areas such as the oil and natural gas industry, the steel industry, energy production and many others. This paper aims to validate the numerical simulation of a laminar flow air flow through the ANSYS CFX program. The numerical investigation reveals the presence of peaks in regions near the pipe wall, in the axial velocity profile, as predicted in the literature. In general, the results of the simulations show good agreement with several numerical references.
\end{abstract}

Keywords: Entry Region; Validation Process; Laminar Flow, Computational Fluid Dynamics

\section{Introdução}

A hidrodinâmica de escoamentos internos em dutos cilíndricos destaca-se como uma das mais presentes no cotidiano da engenharia, fazendo-se constante em praticamente todos os setores industriais, tal como petróleo e gás natural, celulose, indústria nuclear, dentre diversas outras.

Posta tal importância a demanda por estudos cada vez mais elaborados sobre o comportamento da fluidodinâmica neste tipo de geometria é constante. 


\section{Blucher Proceedings X Encontro Científico de Física Aplicada

A abordagem pode ser feita de forma analítica, experimental e também via simulação numérica. Todavia, resultados analíticos ficam restritos a casos mais simples, tal como os abordados aqui, enquanto as outras duas abordagens tem capacidade de potencial para tratar de configurações complexas, contudo com limitações intrínsecas a cada uma [1].

Mais especificamente, nos estudos de região de entrada é muito comum impor um perfil de velocidade uniforme na entrada na tubulação para analisar os efeitos de parede sobre o perfil de velocidade ao longo da direção axial, tal como explorar conceitos importantes como a Teoria da Camada Limite [2]. Esta teoria, por sua vez, apresenta algumas limitações, que devem ser bem entendidas, afim de gerar soluções consolidadas para esta região do escoamento.

No vigente artigo pretende-se solucionar o escoamento laminar em um duto cilíndrico com entrada uniforme, com auxílio de uma ferramenta de dinâmica dos fluidos computacional (DFC), e efetuar o processo de validação completo da malha numérica, ou seja, na região de entrada e na região desenvolvida no escoamento.

\section{Definição do Problema}

A descrição de um problema hidrodinâmica de escoamento de fluidos pode ser bem posta através das leis conservativas de massa e momentum [8]. Considere então, um escoamento incompressível, em regime laminar e de natureza estacionária. Adotadas tais hipóteses de engenharia as expressões conservativas reduzem-se aos formatos mostrados pelas expressões 1 e 2 .

$$
\begin{gathered}
\nabla \cdot \widetilde{u}=0 \\
\widetilde{u} \cdot \nabla \widetilde{u}=-\frac{1}{\rho} \frac{\partial p}{\partial x}+v \nabla^{2} \widetilde{u}
\end{gathered}
$$

A geração de soluções de natureza analítica ou mesmo numérica é um desafio em relação as equações de Navier Stokes por conta principalmente do termo advectivo do lado esquerdo na equação 2 . Neste termo reside a não linearidade da equação diferencial parcial, tal como fontes de problemas de estabilidade caso a solução seja numérica.

No vigente artigo pretende-se simular numericamente o escoamento em um tubo, cujo esquema representativo pode ser apreciado abaixo na figura 2 , que representa um escoamento de ar padrão a $25^{\circ} \mathrm{C}$, modelado como gás ideal. A constante de parametrização da geometria $\alpha=$ $20 D$.

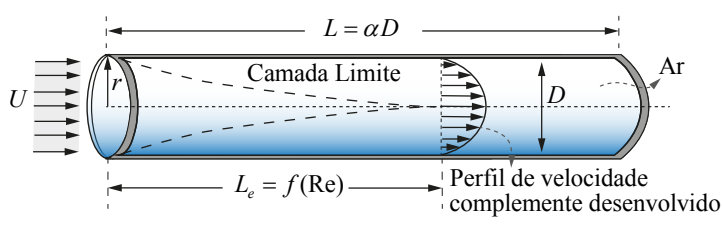

Figura 1: Esquema de Região de Entrada.

É interessante observar que os procedimentos de malha numérica a posteriori dependem da distância de desenvolvimento graficada na figura 2. Tal comprimento depende do adimensional de Reynolds, tal como de várias constantes experimentais, como pode ser visto em [9]. Entretanto, para um primeiro estudo neste artigo será adotada a expressão 3, de natureza mais simples, presente em [11].

$$
\frac{L_{e}}{D} \approx 0,05 R e
$$

\section{Tratamento da Região de Entrada}

As soluções analíticas presentes na literatura que propõe a representação do perfil de velocidade axial na região de entrada são diversas. A solução proposta por Langhaar [3] foi sem dúvidas umas das primeiras e serviu de base para diversas outras pesquisas.

Neste artigo utiliza-se como base analítica de comparação a solução pro- 
posta por Sparrow, Lin e Lundgren [4]. Tal solução baseia-se na lógica de linearizar o termo de convectivo, tornando assim a equação diferencial parcial linear. Esta simplificação tem por consequência direta a validade do princípio da superposição, o que permite uma estrutura de solução tal como na equação 4 .

$$
u=u_{c d}+u^{*}
$$

$\mathrm{Na}$ proposta dos autores assume-se que o perfil de velocidade na região de entrada possa ser determinado pela superposição da parábola de Poiseuille mais a ação de um termo corretor $u^{*}$.

$$
\begin{gathered}
\frac{u}{U}=2\left(1-\eta^{2}\right)+\sum_{i=1}^{\infty} \beta_{i}(\eta) e^{-\alpha_{i}^{2} X *} \\
\beta_{i}(\eta)=\frac{4}{\alpha_{i}^{2}}\left\{\frac{J_{o}\left(\alpha_{i} \eta\right)}{J_{o}\left(\alpha_{i}\right)}-1\right\}
\end{gathered}
$$

A equação 5 consiste numa solução para o perfil de velocidade axial em ambas as regiões do escoamento, pois sua análise limítrofe recai na parábola da região desenvolvida. Algumas variáveis adimensionais importantes para esta solução são postas abaixo, respectivamente a posição radial, posição axial real e posição axial distorcida, na equação 7 . A relação algébrica entre as duas posições axiais pode ser apreciada em [4].

$$
\begin{aligned}
\eta=\frac{r}{r_{o}} \quad X & =\frac{x / r_{o}}{U r_{o} / v} \\
X * & =\frac{x / r_{o}}{U r_{o} / v}
\end{aligned}
$$

\section{Teste de Malha}

No intuito de validar uma malha computacional que não mais influencie nos resultados do problema, executa-se um teste de malha baseado num balanço de forças na região desenvolvida, contabilizado pelo produto $f R e$ [8], e exibido na tabela a seguir.

\begin{tabular}{|l|l|l|}
\hline & C.L & f.Re \\
\hline M1 & - & 51,84 \\
\hline M2 & 2c & 57,28 \\
\hline M3 & 5c & 58,19 \\
\hline M4 & $7 \mathrm{c}$ & 63,68 \\
\hline
\end{tabular}

A tabela acima demonstra um pior resultado na primeira malha, testada sem tratamento de parede, e uma melhora progressiva, assim que a camada-limite é melhor representada. É possível perceber que a quarta malha testada já exibe uma convergência muito grande com a previsão da literatura [2].

Uma segunda maneira de aferir q qualidade da malha pode ser analisada pelo perfil de velocidade na região desenvolvida, mostrado abaixo na figura 4 .

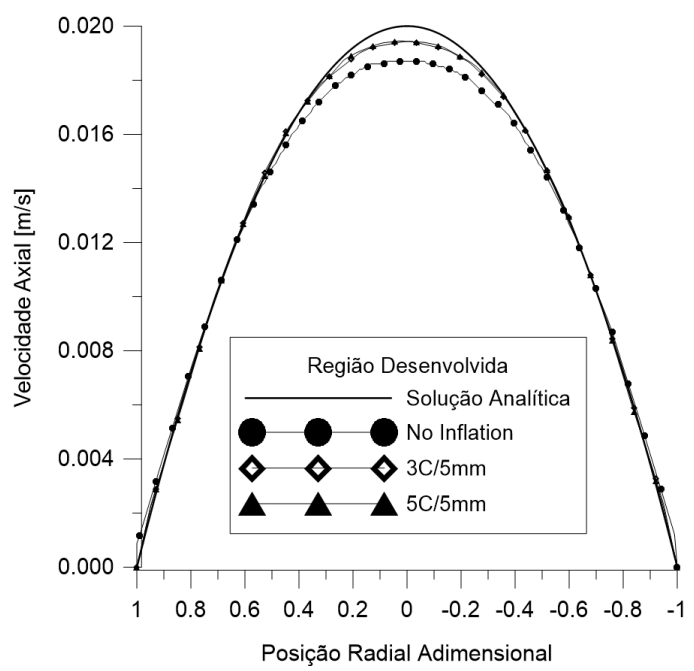

Figura 2: Perfil de Velocidade Desenvolvido.

Os perfis de velocidade trazidos pela figura 4 mostram que um melhor refinamento de parede aproxima as malhas da solução analítica da região desenvolvida. Neste caso, considera-se aqui a malha M3 com cinco camadas de tratamento de parede como satisfatória para a geração dos resultados.

\section{Estudo de Caso}

Uma primeira comparação natural é contrapor os resultados da solução numérica com as predições analíticas para a região de entrada, tal como segue na figura 5. 


\section{Blucher Proceedings X Encontro Científico de Física Aplicada

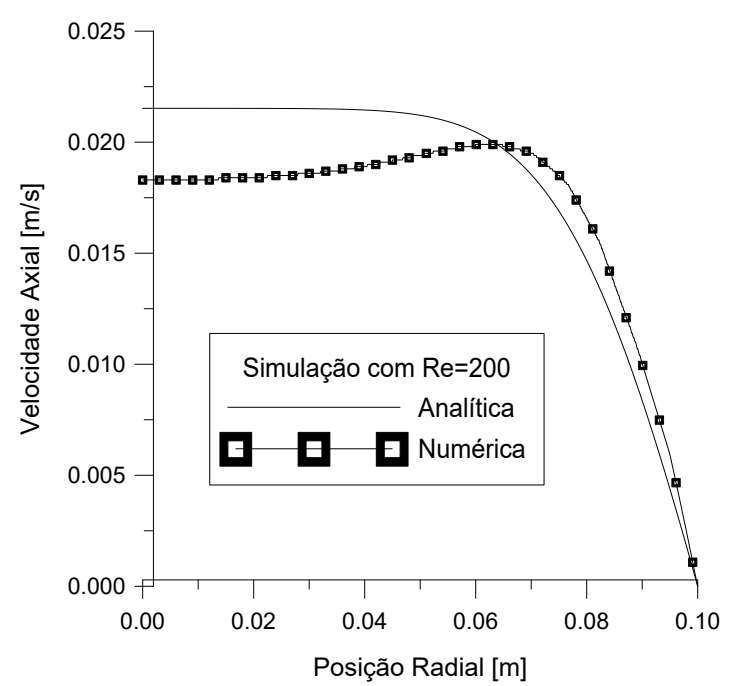

Figura 3: Validação com Solução de Referência.

A discrepância observada entre os resultados é esperada e amplamente relatada na literatura em trabalhos de investigação numérica tal como [13], [6], e [9]. Este comportamento da solução numérica, em primeiro momento, levanta um questionamento quanto a existência física efetiva do fenômeno. Este ponto em aberto foi recentemente solucionado em um artigo experimental, com o uso de velocimetria por ressonância magnética, que comprovou em definitivo a natureza física das curvaturas no perfil de velocidade [12]. Desta forma as previsões geradas neste trabalho pelo programa ANSYS CFX mostram-se alinhadas com a literatura, uma vez que as soluções analíticas fazem uso de premissas simplificadas.

Faz-se interessante também analisar o comportamento dos picos no perfil de velocidade a diferentes números de Reynolds, em uma posição axial fixa de $x=$ $0,5 D$.

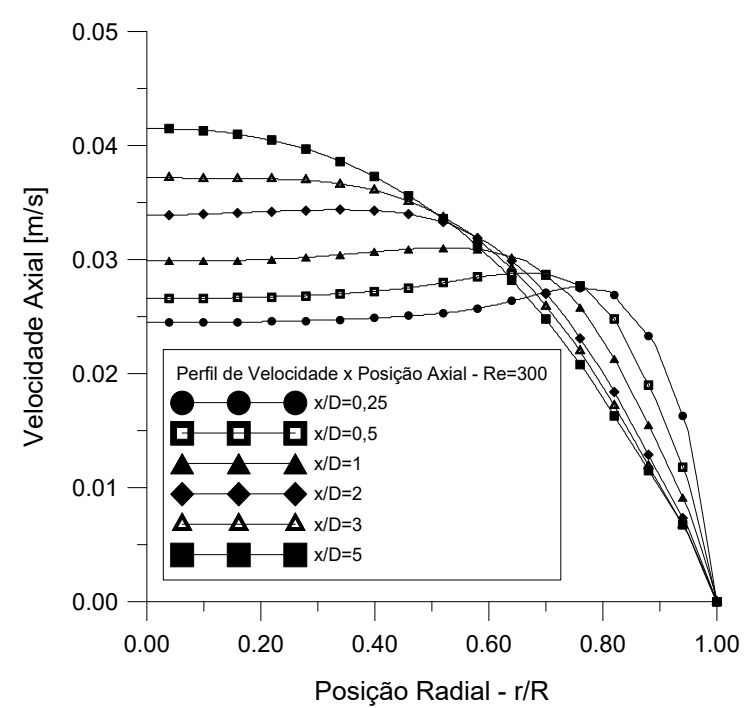

Figura 4: Análise Paramétrica com Re.

Uma observação atenta da figura 5 revela que os pontos de velocidade máxima, para uma mesma posição axial, ocorrem mais próximos a parede com o aumento do número de Reynolds. Esta conclusão apoia-se no fato de que comprimento da região de entrada é alongado proporcionalmente ao aumento do adimensional, conforme a equação 3. Desta forma, para um maior número de $\mathrm{Re}$, a posição axial de $x=0,5 D$ representa uma região menos desenvolvida da camada-limite.

Por fim fixa-se um número de $R e=$ 300 e determina-se numericamente o perfil de velocidade em diversas posições axiais ao longo da região de entrada tal como a seguir.

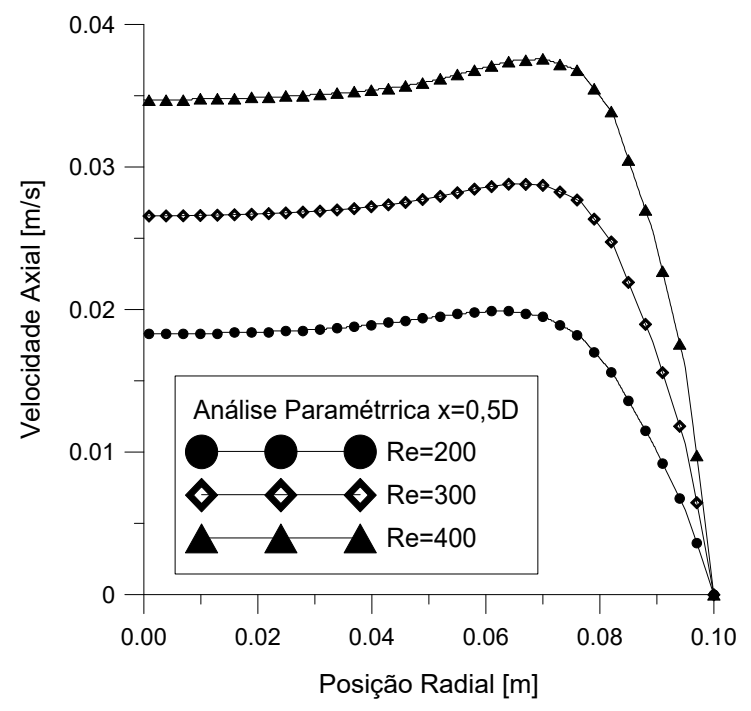

Figura 5: Desenvolvimento do Perfil de Velocidade Axial.

Na figura 5 observa-se um desloca- 


\section{Blucher Proceedings X Encontro Científico de Física Aplicada

mento dos pontos de velocidade máxima da parede para o centro a medida que o escoamento avança. Tal constatação tem alinhamento com o fato de que estes picos surgem por influência da parede. Além do mais, o formato parabólico do perfil da região desenvolvida torna natural o deslocamento dos picos em direção ao centro da tubulação.

\section{Considerações Finais}

A priori pode-se afirmar que a malha foi validada de forma satisfatória, isto posto comprovado pelo pela previsão do balanço de forças de pressão e cisalhantes alinhada com literatura, que confirma tratamento de parede adequado.

As discrepância nos resultados analíticos frente aos numéricos já era esperada, devido a incapacidade da solução analítica de prever os picos. Há alteração no posicionamento radial dos picos com o número de Reynolds para uma mesma posição axial, uma vez que esta movimentação se dá por troca de momento linear, automaticamente atrelada a estrutura do adimensional.

A movimentação radial dos picos da parede em direção ao centro acontece ao longo do desenvolvimento, passando por uma zona axial que se assemelha às soluções analíticas, e logo após recai na região desenvolvida.

Necessita-se mais investigações do fenômeno para compreender a natureza física dos picos, tal como sua ligação com os termos matemáticos da modelagem da dinâmica dos fluidos.

\section{Referências}

[1] FORTUNA, A.O. Técnicas computacionais para dinâminca dos fluidos: conceitos básicos e aplicações. Edusp, 2000.
[2] SCHLICHTING, H.; GERSTEN, K. Boundary-layer theory. Springer, 2016.

[3] LANGHAAR, H.L. Steady flow in the transition length of a straight tube. J. appl. Mech., v. 9, 1942.

[4] SPARROW, E. M.; LIN, S. H.; LUNDGREN, T. S. Flow development in the hydrodynamic entrance region of tubes and ducts. The Physics of Fluids, v. 7, n. 3, p. 338-347, 1964.

[5] FARGIE, D.; MARTIN, B. W. Developing laminar flow in a pipe of circular cross-section. Proceedings of the Royal Society of London. A. Mathematical and Physical Sciences, v. 321, n. 1547, p. 461-476, 1971.

[6] FRIEDMANN, M.; GILLIS, J.; LIRON, N. Laminar flow in a pipe at low and moderate Reynolds numbers. Applied Scientific Research, v. 19, n. 1, p. 426-438, 1968.

[7] SANTOS, Rogério G. dos; FIGUEIREDO, José R. Laminar elliptic flow in the entrance region of tubes. Journal of the Brazilian Society of Mechanical Sciences and Engineering, v. 29, n. 3, p. 233-239, 2007.

[8] BATCHELOR, Cx K.; BATCHELOR, G. K. An introduction to fluid dynamics. Cambridge university press, 1967.

[9] DOMBROWSKI, N. et al. The influence of Reynolds number on the entry length and pressure drop for laminar pipe flow. The Canadian Journal of Chemical Engineering, v. 71, n. 3, p. 472-476, 1993.

[10] OZISIK, M.N. et al. Finite difference methods in heat transfer. CRC Press, 2017. 
[11] KAKAC, S.; SHAH, R.K.; Handbook of single-phase convective heat transfer. 1987.

[12] RECI, A.; SEDERMAN, A.J.; GLADDEN, L.F. Experimental evidence of velocity profile inversion in developing laminar flow using mag- netic resonance velocimetry. Journal of Fluid Mechanics, v. 851, p. 545557, 2018.

[13] ATKINSON, B. et al. Low Reynolds number developing flows. AIChE Journal, v. 15 , n. 4, p. 548-553, 1969. 